\title{
Effect of Bacillus bacteria on activity of pathogen-induced proteins in potato plants and increasing their resistance to Phytophthora infestans (Mont.) de Bary \\ Tsvetkov V.O. ${ }^{2}$, Yarullina L.G. ${ }^{1,2}$, Burkhanova G.F. ${ }^{l}$, Sorokan A.V. ${ }^{l}$
}

${ }^{1}$ Institute of Biochemistry and Genetics, Ufa Federal Research Centre, Ufa, Russiaж ${ }^{2}$ Bashkir State University, Ufa, Russia E-mail: yarullina@bk.ru

Key message. We studied the effect of the Bacillus bacteria on the content and activity of defensive compounds in potato plants upon infection with late blight pathogen. Bacterial treatment had a stimulating effect on the concentration of $\mathrm{H}_{2} \mathrm{O}_{2}$ and transcriptional activity of hydrolase inhibitor genes.

Keywords: potato, Phytophthora infestans, bacteria of the genus Bacillus, ROS, protease and amylase inhibitors

In modern conditions of high anthropogenic pressure on agroecosystems, the issue of ecologically safe crop production, which requires new approaches to protecting plants from pathogens, is acute. Of great importance in the signal regulation of plant resistance to pathogens is assigned to non-pathogenic rhizobacteria that regulate plant growth (plant growth promoting rhizobacteria, PGPR). Most of them trigger a cascade of defense reactions in plant tissues due to the production of various metabolites. Of particular interest among the metabolites of bacteria of the genus Bacillus sp. represent cyclic low molecular weight lipopeptides - surfactin, iturin and fengicin. In particular, surfactins trigger the active generation of $\mathrm{H}_{2} \mathrm{O}_{2}$ in tobacco plants and a number of components of the oxylipin signaling defense system, including the activity of proteinase inhibitors.

Since PGPR positively affects the resistance of plants to pathogens and helps increase their productivity, these compounds are widely used in agricultural practice. At the same time, the mechanisms of the formation of a protective response under the influence of signaling molecules are not well understood.

The object of research was potato plants grown from micro-tubers. Some of the plants were inoculated with a suspension of bacteria Bacillus subtilis and Bacillus thuringiensis, then they were infected with late blight pathogen - oomycete Phytophthora infestans (Mont.) de Bary. A comparative assessment was made of the damage to plants by the pathogen, the content of $\mathrm{H}_{2} \mathrm{O}_{2}$, hydrolases and their inhibitors, and the transcriptional activity of protease and amylase inhibitor genes in plant tissues. Statistical data processing was carried out using computer software StatSoft Statistica 6.0.

Studies have shown that under the influence of treatment with bacteria B. subtilis and B. thuringiensis there is a decrease in the degree of development of symptoms of the disease on potato leaves by an average of $25.0 \pm 2.0 \%$. The data obtained indicate that endophytic bacteria increase the resistance of potato plants. In response to B. subtilis treatment and $P$. infestans infection, the level of $\mathrm{H}_{2} \mathrm{O}_{2}$ in potato leaves increased. Moreover, B. subtilis had a stimulating effect on the production of hydrogen peroxide already 24 hours after infection. Bacteria $B$. thuringiensis exhibited the maximum stimulating effect on the production of $\mathrm{H}_{2} \mathrm{O}_{2} 48$ hours after infection. The formation of ROS is one of the earliest responses of plant cells to contact with a pathogen, as a result of which protective reactions of the plant are induced, including the synthesis of PR proteins.

The main weapon of attack of pathogens are the hydrolytic enzymes produced by them, which destroy the cell walls of plants and ensure the introduction of the fungus into tissues. The protective response of plants is accompanied by the synthesis of inhibitors of these enzymes. In our studies, $B$. subtilis and B. thuringiensis stimulated the transcriptional activity of amylase and proteinase inhibitor genes in uninfected potato plants, but especially when infected. Treatment with $B$. subtilis and $B$. thuringiensis caused an increase in protease activity in the absence of $P$. infestans infection, as well as amylases, both in infected and in uninfected plants. The activity of amylase inhibitors in the leaves of infected plants increased when treated with both species of bacteria; in uninfected plants, treatment with B. thuringiensis resulted in increased activity of protease inhibitors. Probably, in response to infection with $P$. infestans, de novo enzyme inhibitors that can inhibit the pathogen hydrolase activity can be synthesized in potato plants.

The work was partially carried out on the topic of state assignment, No. state. registration AAAA-A16-116020350027-7, and with partial financial support from the Grant of the President of the Russian Federation for state support of young Russian scientists - candidates of sciences, No. 075-15-2019-293. 